\title{
A NEW METHOD FOR THE ACQUISITION OF ULTRASONIC STRAIN IMAGE VOLUMES
}

R. J. Housden, C. Uff, L. Chen, A. H. Gee, G. M. Treece, R. W. Prager and J. C. Bamber CUED/F-INFENG/TR 656

August 2010

University of Cambridge Department of Engineering

Trumpington Street Cambridge CB2 1PZ United Kingdom

Email: rjh80@eng.cam.ac.uk 


\title{
A new method for the acquisition of ultrasonic strain image volumes
}

\author{
${ }^{*}$ R. James Housden, ${ }^{\dagger}$ Christopher Uff, * Lujie Chen, *Andrew H. Gee, \\ ${ }^{*}$ Graham M. Treece, ${ }^{*}$ Richard W. Prager and ${ }^{\dagger}$ Jeffrey C. Bamber \\ *University of Cambridge \\ Department of Engineering \\ Trumpington Street \\ Cambridge CB2 1PZ
}

\begin{abstract}
This paper presents a new method for acquiring 3D volumes of ultrasonic axial strain data. The method uses a mechanically-swept probe to sweep out a single volume while applying a continuously varying axial compression. We examine the image quality and ease of use of the new method with in vitro and in vivo experiments. The new method is easier to use than the current best alternative and so more reliably produces images of superior quality.
\end{abstract}

\section{Introduction}

Ultrasonic strain imaging is an emerging technique for visualising tissue stiffness. Several potential applications have been identified, including the detection of tumours in soft tissue [3, 11, 25], the monitoring of atherosclerosis [7, 23] and the measurement of skin elasticity [27, 6]. This paper is concerned with quasi-static strain imaging [21], which is achieved in 2D by comparing radio-frequency $(\mathrm{RF})$ echo signals from two or more B-scans with a small difference in the axial compression applied through the ultrasound probe. Axial strain is determined by calculating the gradient of axial displacement estimates.

A particular challenge in quasi-static imaging is to achieve a high strain signal to noise ratio in a clinically practical, freehand scan. Individual freehand strain images may have high estimation noise caused by excessive non-axial probe movement or an axial compression magnitude that is either too large or too small. The solution for $2 \mathrm{D}$ imaging is to use an estimate of image quality to identify the best strain data from a sequence of frames with continuously varying compression [14]. This can be used in a quality-weighted averaging process [18] to create a composite strain image. Using this approach, it is currently possible to produce high quality and stable 2D images, requiring no additional equipment to constrain the probe motion.

The $2 \mathrm{D}$ imaging technique has more recently been extended into $3 \mathrm{D}$, offering the potential for more complete visualisation of the anatomy and more accurate measurement of the volume of stiff or soft regions. Currently, there are two categories of techniques for 3D quasi-static strain imaging. The first of these is a direct extension of the $2 \mathrm{D}$ technique in which the probe is moved deliberately in the elevational direction while recording the image sequence. The individual image frames are located in space by a position sensor attached to the probe [16] or, in the case of intravascular imaging, by a continuous pullback method [24]. Provided adjacent B-scans lie within each other's elevational decorrelation range, they produce a sequence of $2 \mathrm{D}$ axial strain images distributed in 3D space. Quality-weighted temporal averaging can be replaced in this case by a quality-weighted 3D spatial filter, which again emphasises the highest quality strain data [18]. We refer to this method as the frame sequence method.

The alternative volume pair approach to 3D acquisition is to record two or more complete volumes of ultrasound data. The volumes can be acquired using either a mechanically-swept 3D probe, or a 2D array probe. There is no change in axial compression while recording each volume, but a small change in compression is applied between them. This compression can be achieved in a laboratory setting using an external fixture to compress the scanning subject $[1,8,10,15,20,22]$ 
or, in the case of ablation imaging, by controlled movement of the ablation electrode [2]. More practically, the probe movement can be applied by hand [26]. Volumes of axial strain data are then obtained by comparing pairs of volumes directly.

Both methods have practical difficulties and require a careful scanning technique to obtain high quality data. At currently achievable volume acquisition rates, the volume pair approach must be used with distinct, individual compressions between volumes and so cannot rely on a continuous axial motion, with quality-weighted averaging, to produce a high quality image. Instead, the user must take care to apply the optimal probe motion, which in clinical practice is ideally applied by hand without any additional equipment to constrain the probe. With the frame sequence approach, it is difficult to ensure adequate elevational frame density over the length of the sequence and there may be low quality regions in the data where the frame density is too sparse. Also, the use of a position sensor can be an inconvenience in a clinical setting.

In this paper, we present in vitro and in vivo evaluations of a variation on the frame sequence approach in which a mechanically-swept 3D probe controls the elevational motion. The probe is moved in a continuous axial motion, in the same way as a freehand $2 \mathrm{D}$ sequence, so that the compression does not need to be precisely controlled. This method therefore avoids the three practical difficulties mentioned above, making it easier to acquire acceptable strain data.

\section{Methods}

The complete strain data acquisition protocol can be divided into three stages: acquisition, motion correction and frame reduction. The acquisition stage makes use of a mechanically-swept 3D probe controlled by an internal stepper motor [26]. We allow the probe to gradually sweep out a volume, pausing at each step of the sweep to record several frames of data. During the recording, the probe is moved continuously up and down by hand in the axial direction, just like in 2D scanning, to vary the compression between individual image frames. Recording several frames at each step increases the chance that there is at least one pair in each location with an appropriate amount of compression for good strain data. For the experiments in this paper, we use five frames per step, which we have found is sufficient for high quality images.

This is followed by an optional motion correction stage. It is possible that the applied axial motion causes the probe to slide laterally or elevationally as the sweep is recorded. Motion correction is applied if necessary to correct the distortion this would cause in the 3D images. If correction is required, then the acquisition stage records a second sweep immediately after and overlaid on the first, as shown in Figure 1(a). This reference sweep is recorded very quickly with no deliberate probe motion and is therefore assumed to be an undistorted representation of the scanned subject. Individual frames from the strain sweep are registered in turn to this sweep (Figure 1(b)), giving their location relative to a fixed reference.

Registration of a frame to the reference sweep is achieved by matching a grid of small 2D windows of scan converted B-mode image data. Individual windows are matched in a $3 \mathrm{D}$ search within the reference sweep, using a multiscale exhaustive search for the peak correlation value (as used in [4]). Displacement continuity is enforced within the frame by using a quality guided matching process [5]. The window location estimates are then processed by a Random Sample Consensus (RANSAC) algorithm [9] to exclude outliers, further enforcing continuity. The robustness provided by the RANSAC algorithm is particularly important to maintain accuracy in the elevational direction, where the 2D matching windows cannot easily distinguish the best matching reference frame from adjacent frames. The final sequence of frame positions is refined using a nonparametric regularisation filter [12]. This smoothing process enforces some inter-frame continuity in the frame positions, which would otherwise be positioned independently from surrounding frames.

Finally, the frame reduction step identifies the highest quality pair of frames at each motor step. The remaining frames are then discarded. The highest quality data in each step are usually confined to one of the frame pairs, with probe motion closest to the ideal compression. This optional stage of processing involves calculating a $2 \mathrm{D}$ strain image for each pair of image frames 


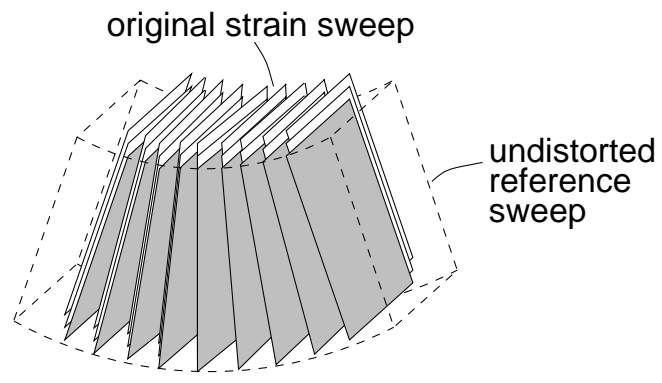

(a)

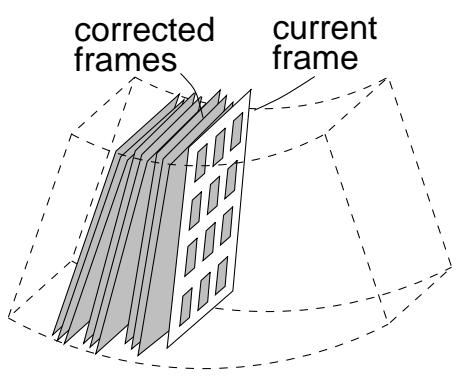

(b)

Figure 1: Motion correction to a reference sweep. (a) Lateral and elevational motion in the strain sweep is corrected by registering to an undistorted reference sweep overlaid on the strain sweep. (b) Registration is achieved by considering each frame individually in turn. The figure shows a situation part way through the motion correction process in which some frames have been corrected. The current frame is located by matching small patches of data from the frame to the reference sweep.

and comparing some quality metric for the whole frame to identify the best pair. Various framewise quality metrics could be used; we have chosen to use the average value of multiple strain $\mathrm{SNR}_{e}$ measurements over each 2D strain image. $\mathrm{SNR}_{e}$ is defined as $\bar{s} / \sigma_{s}$, where $\bar{s}$ is the mean strain and $\sigma_{s}$ is the standard deviation calculated over small $(3 \times 3)$ kernels of strain estimation windows.

Once the data are recorded, with or without motion correction and frame reduction, the volumetric strain images are calculated in the same way as for a freehand 3D frame sequence. Each adjacent frame pair is compared in the usual way to produce a $2 \mathrm{D}$ axial strain image. Frame pairs between motor steps are excluded, because they may be separated too far elevationally to provide useful data. Details of our method for $2 \mathrm{D}$ axial strain calculation can be found in our earlier publications $[16,17,18,19]$. The final stage of processing is the quality weighted spatial filter, which uses a 3D Gaussian smoothing kernel, with each sample weighted by the precision of the strain estimate [18], to generate a volume of data from the sequence of $2 \mathrm{D}$ images.

\section{Experiments and results}

\subsection{Experimental setup}

The effectiveness of the new technique was examined through in vitro experiments and in vivo scans. The scanning system was a GE RSP6-12 mechanically-swept probe (GE Healthcare, Chalfont St Giles, UK) interfaced to a Dynamic Imaging Diasus ultrasound scanner. The system was set to sweep out between 15 and 20 degrees, varying between data sets, in 75-100 steps, producing sweeps of 375-500 frames. In all cases, the elevational spacing between steps was $0.35 \mathrm{~mm}$ at a depth of $2 \mathrm{~cm}$. Each frame of RF data comprised 127 A-lines covering a width of $2.5 \mathrm{~cm}$ and sampled at $66.67 \mathrm{MHz}$ to a depth of $3.5 \mathrm{~cm}$. The envelope and phase of the RF data were calculated using matched Hilbert filters and formed the basis of all strain calculations.

Where the optional motion correction was applied, the reference sweep size was chosen automatically such that it was 1.5 times longer than the strain sweep, within the constraint of the motor's maximum range of 29 degrees. The number of reference steps was chosen to give an average elevational frame spacing of $0.15 \mathrm{~mm}$ at the frame centre. Motion correction calculations were based on scan converted images, derived from the envelope of the RF data, with a scale of $0.10 \mathrm{~mm} /$ pixel. For verification of the motion correction, we acquired some data sets with a Northern Digital Polaris optical tracking system (NDI, Waterloo, Ontario, Canada) to record the location of the probe as each image frame was acquired. Typical results of motion correction are 
shown in Figure 2.

Our various in vitro experiments made use of two phantoms. The first was an elasticity quality assurance (QA) phantom (Model 049, CIRS, Norfolk, Virginia, USA). The phantom contains $10 \mathrm{~mm}$ diameter inclusions of various stiffnesses above and below that of the background material, located at a depth of $15 \mathrm{~mm}$. It has a flat surface, making it a relatively easy subject to scan. Since we are particularly interested in the ease of use of the new method, we also recorded some data on a breast biopsy phantom (Model 052, CIRS) containing stiff and soft inclusions of various sizes. This has a more realistic surface shape and so presents more of a challenge for scanning, particularly for large footprint probes like the RSP6-12. Finally, we obtained several in vivo scans using both our new method and the volume pair method for comparison. These comprised several musculoskeletal scans recorded in our laboratory, as well as scans of the human brain obtained intra-operatively at the Royal Free Hospital, London ${ }^{1}$.

\subsection{Comparison with other methods}

We are interested in how the new method compares with existing 3D strain imaging methods, in terms of both achievable image quality and ease of use. In our earlier comparison of the frame sequence and volume pair methods [13], we found that the volume pair approach was preferable. Here we compare the new method to the volume pair method. Full details of the volume pair acquisition and processing are given in [26]. The volume pair scans were of the same size as those for the new method, in terms of number of motor steps and swept angle, and imaged the same location on the scanning subjects. Comparisons were made with the new method using all recorded frames and after reducing to the best frame-pair in each step.

Figure 3 shows a selection of strain images comparing the two methods. One of the advantages of the volume pair method is its ability to use 3D displacement estimation windows, which improve the $\mathrm{SNR}_{e}$ by including more data, but worsen the elevational resolution. In order to make a fair comparison, with approximately equivalent resolution, the elevational size of the Gaussian spatial filter was reduced to compensate for the resolution penalty of using $3 \mathrm{D}$ windows.

As a quantitative measure of image quality, we used the average of multiple $\mathrm{SNR}_{e}$ values calculated using $1.5 \mathrm{~mm}$ kernels within a region of interest in the 3D strain images. This approach assumes that while the strain may vary over the image, the true strain is approximately constant within the small kernel, and any variations affecting the $\mathrm{SNR}_{e}$ estimate are due to strain estimation noise. For the phantom scans, the region of interest was typically a spherical region centred on an inclusion but excluding the inclusion itself in order to have uniform stiffness within the region of interest. For the in vivo data, regions of interest were specified by defining the centre and radius of a sphere taking particular care to outline the same part of the anatomy in the B-mode image data acquired by the two scanning methods. As far as is possible in in vivo data, these regions of interest were defined on parts of the data with approximately uniform stiffness. Figure 3 shows some typical regions of interest and Table 1 shows the results of this comparison for all data sets.

\section{Discussion}

The results in Table 1 show that in the majority of cases, the new method outperforms the volume pair method for image quality. This superior quality is visible in Figure 3(a) where the background grey level appears more uniform using the new method and also in Figure 3(b) where the textured noise mask extends further up the image for the volume pair result, indicating lower quality data. As expected, the optional frame reduction feature of the new method, which creates a data set of the same number of frames as the volume pair method, has little effect on the $\mathrm{SNR}_{e}$ compared

\footnotetext{
${ }^{1}$ Our scanning protocol for the laboratory scans has been reviewed by a local ethics committee and informed consent of the subjects was obtained. Full ethical approval for the clinical scans was obtained from the National Hospital for Neurology and Neurosurgery and the Institute of Neurology Joint Regional Ethics Committee (reference 08/H0716/92). Research and development approval and sponsorship was obtained from the Royal Free Hospital Clinical R\&D Department (reference 7745).
} 


\section{(a) QA phantom data 1}

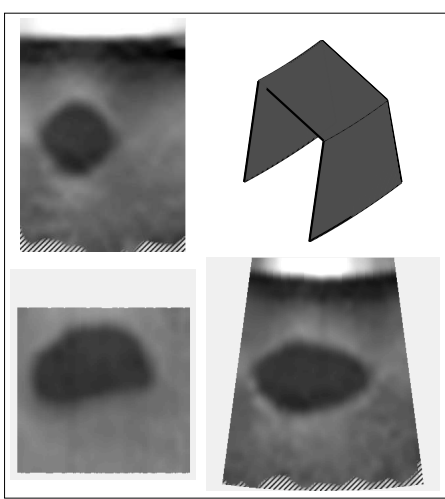

(a1) uncorrected

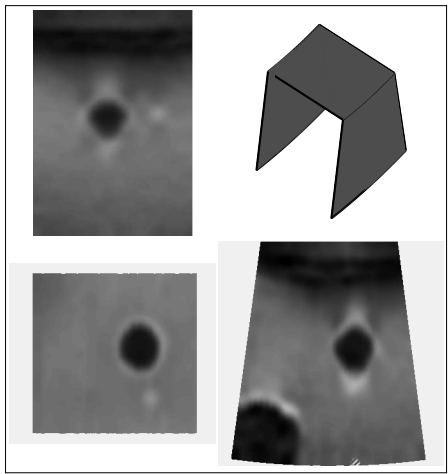

(b1) uncorrected

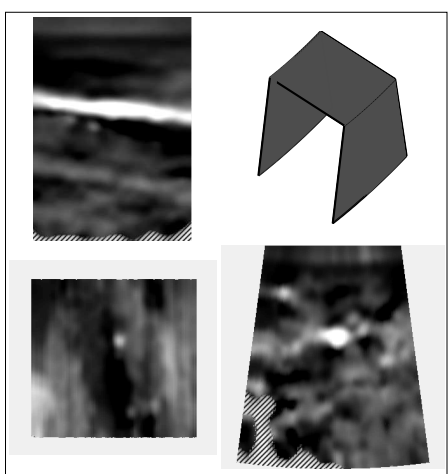

(c1) uncorrected

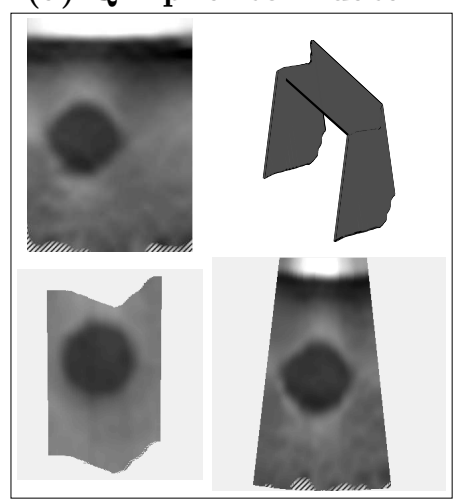

(a2) corrected

(b) biopsy phantom data 1

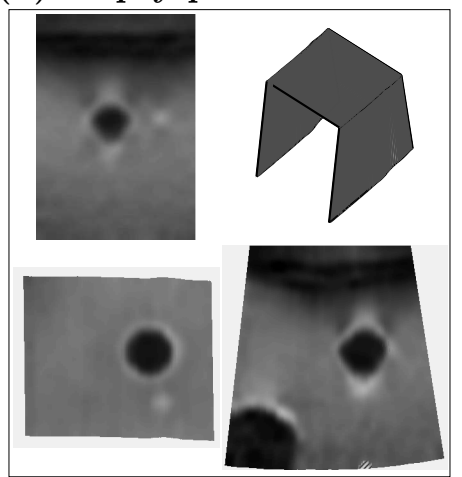

(b2) corrected

(c) forearm data 1

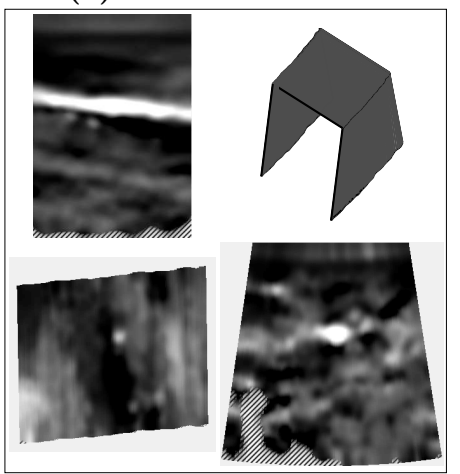

(c2) corrected

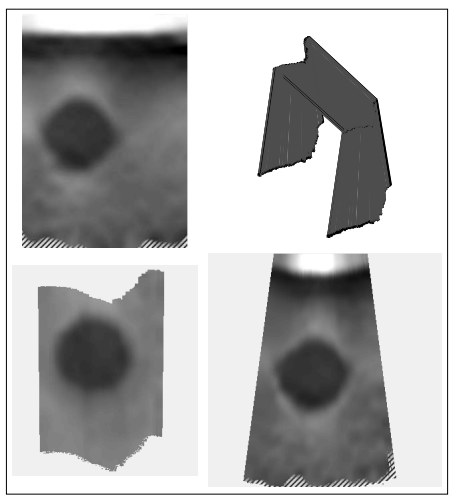

(a3) position sensor

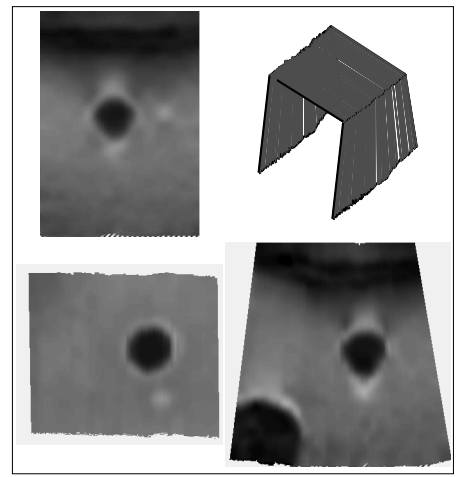

(b3) position sensor

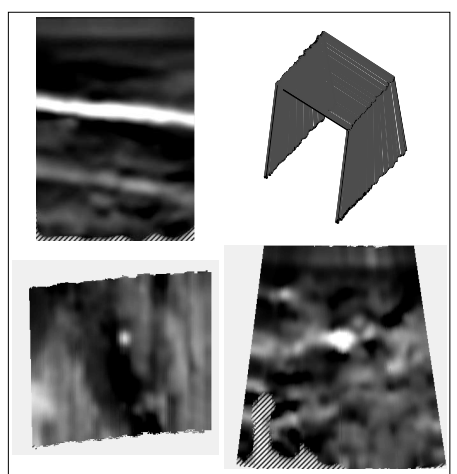

(c3) position sensor

Figure 2: Examples of motion correction. (a) An example of motion correction in which the probe was deliberately moved a large distance laterally and elevationally during the scan, in order to highlight the effect of probe motion. Results are shown before and after motion correction and also with the frames located using a position sensor. In each result, the top left image is an axial-lateral view showing a single strain image from the sequence, the lower left is a lateralelevational slice through the volume and the lower right is an axial-elevational slice. The top right image is an outline view, showing the location of each image frame. The strain images show stiff regions as dark and soft regions as light shades of grey. The textured pattern at the bottom of the data is a feature of our strain imaging algorithm [18] which masks regions where the quality falls below a certain threshold. (b-c) More realistic examples with no deliberate lateral or elevational movement. 
(a) biopsy phantom data 4

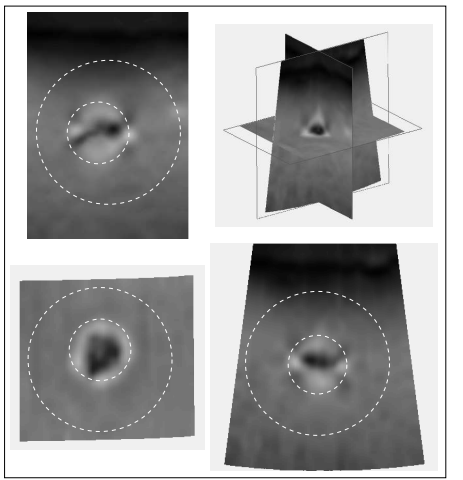

(a1) all frames

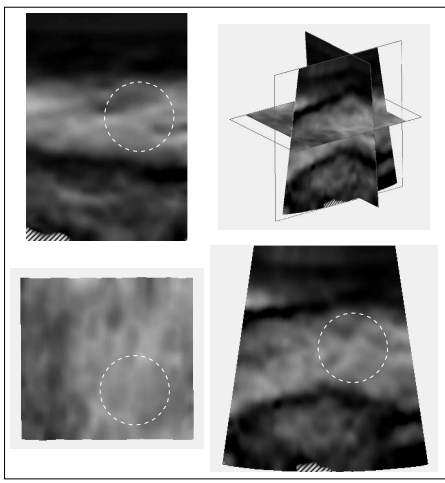

(b1) all frames

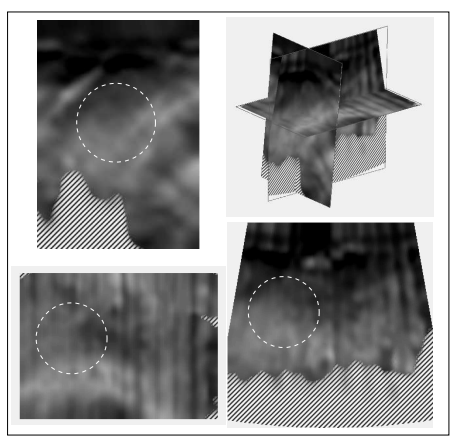

(c1) all frames

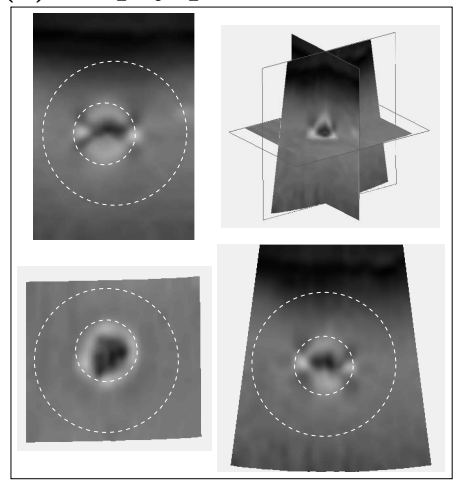

(a2) best pairs

(b) calf muscle data 2

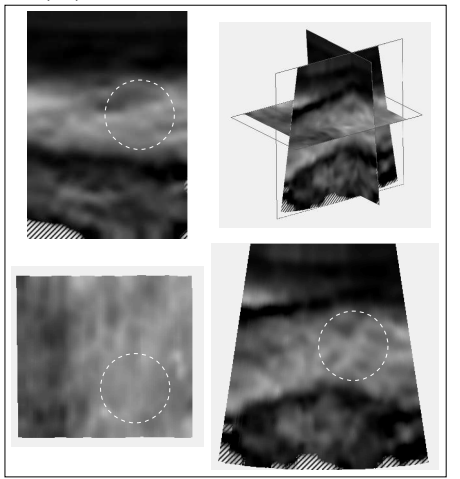

(b2) best pairs

(c) brain data 1

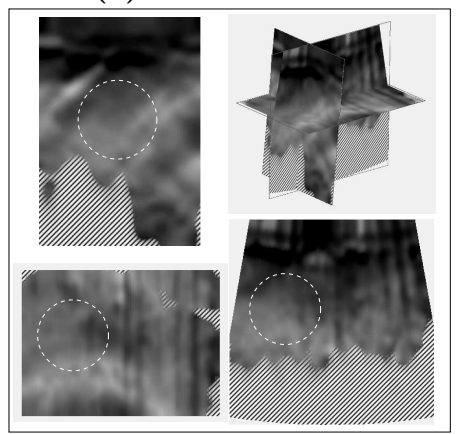

(c2) best pairs

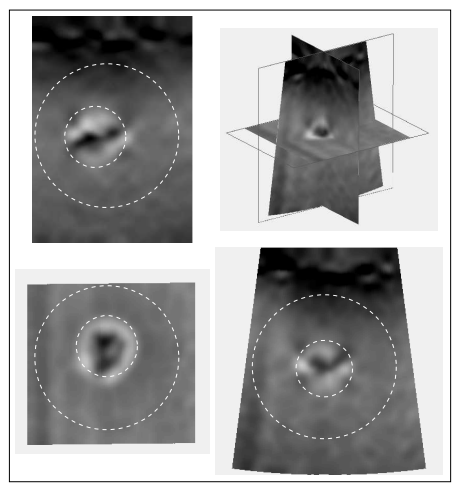

(a3) volume pair

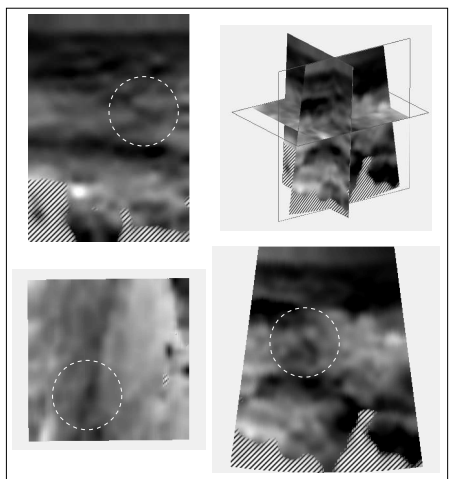

(b3) volume pair

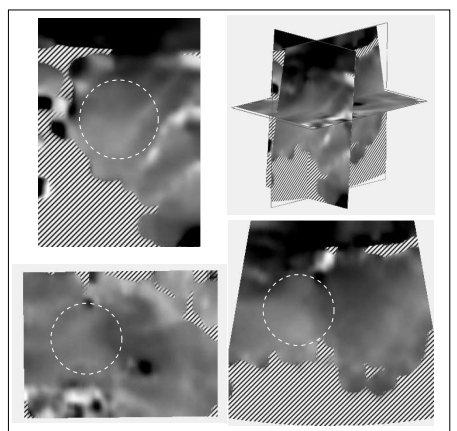

(c3) volume pair

Figure 3: Image quality comparison. The figure shows results comparing image quality for the new method using all five frames at each step, using only the best pair at each step, and the volume pair method. The top right image in each subfigure is a 3D view showing three orthogonal slices through the data. The other views are the same as in Figure 2. The white dashed lines show the regions used to calculate $\mathrm{SNR}_{e}$ values for Table 1 . In the phantom data sets, the $\mathrm{SNR}_{e}$ calculation considered only data from outside the inclusions, as shown in (a). The inclusion in (a) is a cyst-like region with low RF signal strength and so produces unreliable strain values. 


\begin{tabular}{r|c|c|c}
\hline data & \multicolumn{3}{|c}{ SNR $_{e}$} \\
\cline { 2 - 4 } & all frames & best pairs & volume pair \\
\hline QA phant. 2 & $\mathbf{3 4 . 0 7}$ & 33.43 & 22.22 \\
QA phant. 3 & $\mathbf{4 7 . 1 7}$ & 46.56 & 29.69 \\
QA phant. 4 & $\mathbf{7 5 . 9 4}$ & 73.58 & 42.32 \\
QA phant. 5 & $\mathbf{6 0 . 2 8}$ & 59.60 & 38.39 \\
\hline biopsy phant. 1 & $\mathbf{4 0 . 9 0}$ & 35.59 & 39.45 \\
biopsy phant. 2 & 41.62 & $\mathbf{4 5 . 9 4}$ & 28.95 \\
biopsy phant. 3 & $\mathbf{4 3 . 9 6}$ & 43.19 & 31.47 \\
biopsy phant. 4 & 39.28 & $\mathbf{4 4 . 0 7}$ & 30.50 \\
biopsy phant. 5 & 47.76 & 49.81 & $\mathbf{5 5 . 6 6}$ \\
\hline calf muscle 1 & 11.12 & $\mathbf{1 1 . 3 2}$ & 8.65 \\
calf muscle 2 & $\mathbf{1 7 . 4 0}$ & 17.07 & 8.60 \\
upper arm 1 & 9.75 & $\mathbf{1 0 . 1 4}$ & 4.97 \\
upper arm 2 & 7.07 & $\mathbf{7 . 0 8}$ & 6.14 \\
upper arm 3 & 5.53 & 5.58 & $\mathbf{6 . 1 4}$ \\
\hline brain 1 & 14.75 & 15.72 & $\mathbf{2 0 . 9 0}$ \\
brain 2 & 8.35 & 8.68 & $\mathbf{1 4 . 6 0}$ \\
brain 3 & 11.37 & 11.49 & $\mathbf{1 7 . 5 3}$ \\
\hline
\end{tabular}

Table 1: $\mathbf{S N R}_{e}$ comparison. The table shows $\mathrm{SNR}_{e}$ values for the different scanning and processing methods. The largest value in each case is highlighted. The values shown are averages of multiple $\mathrm{SNR}_{e}$ values calculated from $1.5 \mathrm{~mm}$ kernels in a spherical region of interest in the strain images. Figure 3 shows examples of the regions used. Certain data sets were not used for the $\mathrm{SNR}_{e}$ comparison. Those with deliberate large motions were excluded, because significant changes in frame density due to elevational motion could affect the image quality. The forearm 1 data set was not used because a corresponding region could not be found in the two scans. All data sets were subjected to motion correction except for the brain data, which were acquired with a careful scanning technique.

to using all the frames. This is therefore a useful way to reduce processing and data storage requirements.

A few data sets, particularly the brain data, have a higher $\mathrm{SNR}_{e}$ with the volume pair method. This can be explained by two effects. With a very carefully controlled scanning motion, as was used for the brain data, it is possible to record high quality data with the volume pair protocol. Also, there is an artefact specific to the new acquisition method, demonstrated in Figure 3(c), that can reduce its $\mathrm{SNR}_{e}$. The axial-lateral views appear to be of high quality. However, the lateralelevational and axial-elevational views for the new method show a banded effect in the elevational direction. This is caused by the method's reliance on a separate 2D normalisation [18] for each frame to compensate for variations in the applied compression, and is more obvious when there is a sparsity of high quality data in the sequence. In comparison, the volume pair method applies the same compression and 3D normalisation to the whole volume and so does not experience this variation. Notwithstanding this, all data sets recorded using the new method have produced useful strain images.

The benefits of the optional motion correction step are seen in Figure 2. The QA phantom data set (Fig. 2(a)) is a scan of a spherical inclusion, and the image for the uncorrected data is severely distorted by probe motion. After motion correction, the inclusion appears more spherical, and the image more closely matches that obtained using the position sensor. The biopsy phantom and in vivo data (Figs. 2(b) and (c)) give similar results, with the motion corrected images more closely following the position sensor. The examples in Figures 2(b) and (c), in which the data were not deliberately distorted, are typical of the amount of probe movement experienced in the scans in this paper. It is of course the case that with a less careful scanning technique there is likely to be more lateral or elevational probe movement. Motion correction is therefore a useful 
step that relaxes the constraints on the probe motion, allowing the user to acquire data with a less carefully controlled scanning technique. Also, in scans requiring only a qualitatively correct view of the anatomy, motion correction may not be necessary, but when quantitative measurements such as inclusion volume are required, or if the shape of image features is important, then motion correction is able to reduce the distortion of the image and provide more accurate measurements.

Considering acquisition and processing times, the new method takes more time than the volume pair method. For the 375-frame sequences with $3.5 \mathrm{~cm}$ depth used in this paper, the new method requires $15-20$ seconds to record the data, whereas the volume pair method needs approximately 2 seconds per volume. However, the volume pair method often requires that several volumes are acquired in order to find a good pair, particularly with in vivo data. For processing times, running single threaded on a $2.4 \mathrm{GHz}$ Quad Core CPU, motion correction takes around 30 seconds, and strain processing for the new method takes approximately 45 seconds. The volume pair method is significantly faster, requiring only 10 seconds to calculate a volume of strain data.

In terms of practical ease of use, our experience has been that the new method is easier than the volume pair method. Its major advantage is the use of quality-based frame selection from multiple frame pairs, automatically selecting the best data from the continuously varying motion. There is therefore a range of possible scanning motions that will produce useful data. Consequently, all of the data recorded for this paper using the new method produced acceptable strain images at the first attempt. In comparison, the volume pair method admits only a small range of acceptable motions, and the user is required to carefully control the motion in order to record useful data. It is often necessary, particularly with in vivo data, to make several attempts at recording volume pair data before an acceptable image quality is achieved. Despite the longer acquisition times, the ease of use of our new method makes it an attractive alternative, allowing the acquisition of high quality $3 \mathrm{D}$ axial strain data with ease.

\section{Conclusions}

We have presented a new method for recording volumes of axial strain data and have shown that it produces images of comparable and often higher quality than the volume pair method. This is largely because of quality-based selection of strain data from multiple image pairs acquired with a continuously varying compression technique. As this is the same technique required for $2 \mathrm{D}$ scanning, it makes an attractive option for clinically practical volumetric strain imaging.

\section{References}

[1] S. I. Awad and J. T. Yen. 3-D strain imaging using a sparse rectilinear 2D array. In IEEE Ultrasonics Symposium, 200\%, pages 228-231, New York, USA, October 2007.

[2] S. Bharat, T. G. Fisher, T. Varghese, T. J. Hall, J. Jiang, J. A. Madsen, E. L. Zagzebski, and F. T. Lee Jr. Three-dimensional electrode displacement elastography using the Siemens C7F2 fourSight four-dimensional ultrasound transducer. Ultrasound in Medicine and Biology, 34(8):1307-1316, August 2008.

[3] E. S. Burnside, T. J. Hall, A. M. Sommer, G. K. Hesley, G. A. Sisney, W. E. Svensson, J. P. Fine, J. Jiang, and N. J. Hangiandreou. Differentiating benign from malignant solid breast masses with US strain imaging. Radiology, 245(2):401-410, November 2007.

[4] L. Chen, R. J. Housden, G. M. Treece, A. H. Gee, and R. W. Prager. A hybrid displacement estimation method for ultrasonic elasticity imaging. IEEE Transactions on Ultrasonics, Ferroelectrics, and Frequency Control, 57(4):866-882, April 2010.

[5] L. Chen, G. M. Treece, J. E. Lindop, A. G. Gee, and R. W. Prager. A quality-guided displacement tracking algorithm for ultrasonic elasticity imaging. Medical Image Analysis, 13(2):286-296, April 2009. 
[6] L. Coutts, J. Bamber, N. Miller, and P. Mortimer. Ultrasound elastography of the skin and subcutis under surface extensive loading. Ultrasound, 14(3):161-166, August 2006.

[7] C. L. de Korte, A. H. W. van der Steen, E. I. Céspedes, G. Pasterkamp, S. G. Carlier, F. Mastik, A. H. Schoneveld, P. W. Serruys, and N. Bom. Characterization of plaque components and vulnerability with intravascular ultrasound elastography. Physics in Medicine and Biology, 45(6):1465-1475, June 2000.

[8] J-F. Deprez, E. Brusseau, C. Schmitt, G. Cloutier, and O. Basset. 3D estimation of soft biological tissue deformation from radio-frequency ultrasound volume acquisitiond. Medical Image Analysis, 13(1):116-127, February 2009.

[9] M. A. Fischler and R. C. Bolles. Random sample consensus: a paradigm for model fitting with applications to image analysis and automated cartography. Communications of the ACM, 24(6):381-395, June 1981.

[10] T. G. Fisher, T. J. Hall, S. Panda, M. S. Richards, P. E. Barbone, J. Jiang, J. Resnick, and S. Barnes. Volumetric elasticity imaging with a 2-D CMUT array. Ultrasound in Medicine and Biology, 36(6):978-990, June 2010.

[11] B. S. Garra, E. I. Cespedes, J. Ophir, S. R. Sprat, R. A. Zuurbier, C. M. Magnant, and M. F. Pennanen. Elastography of breast lesions: initial clinical results. Radiology, 202(1):79-86, January 1997.

[12] P. J. Green and B. W. Silverman. Nonparametric regression and generalized linear models: A roughness penalty approach. CRC Press, 1993.

[13] R. J. Housden, A. H. Gee, G. M. Treece, and R. W. Prager. 3-D ultrasonic strain imaging using freehand scanning and a mechanically-swept probe. IEEE Transactions on Ultrasonics, Ferroelectrics, and Frequency Control, 57(2):501-506, February 2010.

[14] J. Jiang, T. J. Hall, and A. M. Sommer. A novel image formation method for ultrasonic strain imaging. Ultrasound in Medicine and Biology, 33(4):643-652, April 2007.

[15] M. Krueger, A. Pesavento, H. Ermert, K. M. Hiltawsky, L. Heuser, H. Rosenthal, and A. Jensen. Ultrasonic strain imaging of the female breast using phase root seeking and three-dimensional "optical flow". In Proceedings of the IEEE Ultrasonics Symposium, 1998, volume 2, pages 1757-1760, Sendai, Japan, October 1998.

[16] J. E. Lindop, G. M. Treece, A. H. Gee, and R. W. Prager. 3D elastography using freehand ultrasound. Ultrasound in Medicine and Biology, 32(4):529-545, April 2006.

[17] J. E. Lindop, G. M. Treece, A. H. Gee, and R. W. Prager. Estimation of displacement location for enhanced strain imaging. IEEE Transactions on Ultrasonics, Ferroelectrics, and Frequency Control, 54(9):1751-1771, September 2007.

[18] J. E. Lindop, G. M. Treece, A. H. Gee, and R. W. Prager. An intelligent interface for freehand strain imaging. Ultrasound in Medicine and Biology, 34(7):1117-1128, July 2008.

[19] J. E. Lindop, G. M. Treece, A. H. Gee, and R. W. Prager. Phase-based ultrasonic deformation estimation. IEEE transactions on Ultrasonics, Ferroelectrics, and Frequency Control, 55(1):94-111, January 2008.

[20] A. Lorenz, A. Pesavento, M. Pesavento, and H. Ermert. Three-dimensional strain imaging and related strain artifacts using an ultrasonic 3D abdominal probe. In Proceedings of the IEEE Ultrasonics Symposium, 1999, volume 2, pages 1657-1660, Caesars Tahoe, NV, USA, October 1999. 
[21] J. Ophir, I. Céspedes, H. Ponnekanti, Y. Yazdi, and X. Li. Elastography: a quantitative method for imaging the elasticity of biological tissues. Ultrasonic Imaging, 13(2):111-134, April 1991.

[22] M. S. Richards, P. E. Barbone, and A. A. Oberai. Quantitative three-dimensional elasticity imaging from quasi-static deformation: a phantom study. Physics in Medicine and Biology, 54(3):757-779, February 2009.

[23] J. A. Schaar, C. L. de Korte, F. Mastik, C. Strijder, G. Pasterkamp, E. Boersma, and P. W. Serruys. Characterizing vulnerable plaque features with intravascular elastography. Circulation, 108(21):2636-2641, November 2003.

[24] J. A. Schaar, C. L. de Korte, F. Mastik, L. C. A. van Damme, R. Krams, P. W. Serruys, and A. F. W. van der Steen. Three-dimensional palpography of human coronary arteries. Ex vivo validation and in-patient evaluation. Herz, 30(2):125-133, February 2005.

[25] R. Souchon, O. Rouvière, A. Gelet, V. Detti, S. Srinivasan, J. Ophir, and J-Y. Chapelon. Visualisation of HIFU lesions using elastography of the human prostate in vivo: preliminary results. Ultrasound in Medicine and Biology, 29(7):1007-1015, July 2003.

[26] G. M. Treece, J. E. Lindop, A. H. Gee, and R. W. Prager. Freehand ultrasound elastography with a 3-D probe. Ultrasound in Medicine and Biology, 34(3):463-474, March 2008.

[27] M. Vogt and H. Ermert. Development and evaluation of a high-frequency ultrasound-based system for in vivo strain imaging of the skin. IEEE Transactions on Ultrasonics, Ferroelectrics, and Frequency Control, 52(3):375-385, March 2005. 